\title{
On the Decay of Crossing Numbers
}

\author{
Jacob Fox ${ }^{1}$ and Csaba D. Tóth ${ }^{2}$ \\ ${ }^{1}$ Department of Mathematics, Princeton University, Princeton, NJ \\ licht@mit.edu \\ ${ }^{2}$ Department of Mathematics, Massachusetts Institute of Technology, Cambridge, MA \\ toth@math.mit.edu
}

\begin{abstract}
The crossing number $\operatorname{cr}(G)$ of a graph $G$ is the minimum number of crossings over all drawings of $G$ in the plane. In 1993, Richter and Thomassen RT93 conjectured that there is a constant $c$ such that every graph $G$ with crossing number $k$ has an edge $e$ such that $\operatorname{cr}(G-$ $e) \geq k-c \sqrt{k}$. They showed only that $G$ always has an edge $e$ with $\operatorname{cr}(G-e) \geq \frac{2}{5} \operatorname{cr}(G)-O(1)$. We prove that for every fixed $\epsilon>0$, there is a constant $n_{0}$ depending on $\epsilon$ such that if $G$ is a graph with $n>n_{0}$ vertices and $m>n^{1+\epsilon}$ edges, then $G$ has a subgraph $G^{\prime}$ with at most $\left(1-\frac{1}{24 \epsilon}\right) m$ edges such that $\operatorname{cr}\left(G^{\prime}\right) \geq\left(\frac{1}{28}-o(1)\right) \operatorname{cr}(G)$.
\end{abstract}

\section{Introduction}

The crossing number $\operatorname{cr}(G)$ of a (simple) graph $G$ is the minimum possible number of crossings in any drawing of $G$ in the plane. A famous result of Ajtai et al. ACNS82 and Leighton [L84] states that if $G$ is a graph with $n$ vertices and $m \geq 4 n$ edges, then

$$
\operatorname{cr}(G) \geq \frac{m^{3}}{64 n^{2}} .
$$

For graphs with $n$ vertices and $m \geq \frac{103}{16} n$ edges, Pach et al. PRTT04] improved Inequality (11) by a constant factor to

$$
\operatorname{cr}(G) \geq \frac{1024}{31827} \frac{m^{3}}{n^{2}}
$$

It is well known that for every positive integer $k$, there is a graph $G$ and an edge $e$ of $G$ such that $\operatorname{cr}(G)=k$ but $G-e$ is planar. In 1993, Richter and Thomassen RT93 conjectured that there is a constant $c$ such that for every nonempty graph $G$ with crossing number $k$, there is an edge $e$ of $G$ such that $\operatorname{cr}(G-e) \geq k-c \sqrt{k}$. They showed only that $G$ always has an edge $e$ with $\operatorname{cr}(G-e) \geq \frac{2}{5} \operatorname{cr}(G)-O(1)$. Salazar $[\mathrm{S} 00$ ] proved that for every graph $G$ with no vertices of degree 3, there is an edge $e$ of $G$ such that $\operatorname{cr}(G-e) \geq \frac{1}{2} \operatorname{cr}(G)-O(1)$. Pach and G. Tóth [PT00] showed for every connected graph $G$ with $n$ vertices, $m \geq 1$ edges, and every edge $e$ of $G$, that the decay is bounded by

$$
\operatorname{cr}(G-e) \geq \operatorname{cr}(G)-m+1 \text {. }
$$


This, combined with Inequality (2), is better than Richter-Thomassen's bound for graphs with $n$ vertices and $m \geq 8.1 n$ edges. By Inequality (1), it also confirms the Richter-Thomassen conjecture for dense graphs, that is, for graphs with $\Omega\left(n^{2}\right)$ vertices.

In this paper, we show that from every graph $G$ that is not too sparse, we can delete a constant fraction of the edges such that the the crossing number of the remaining subgraph $G^{\prime}$ is at least a constant fraction of the crossing number of $G$.

Theorem 1. For every $\epsilon>0$, there is a constant $n_{0}$ depending on $\epsilon$ such that if $G$ is a graph with $n>n_{0}$ vertices and $m>n^{1+\epsilon}$ edges, then $G$ has a subgraph $G^{\prime}$ formed by deleting at least $\epsilon m / 24$ edges from $G$ such that

$$
\operatorname{cr}\left(G^{\prime}\right) \geq\left(\frac{1}{28}-o(1)\right) \operatorname{cr}(G) .
$$

To prove Theorem 1, we derive in Sections 3 and 4 new lower bounds on the crossing number that improve on Inequality (1) for graphs with highly irregular degree patterns.

\section{Drawing Edges with the Embedding Method}

We use the embedding method along the lines of Leighton L83, Richter and Thomassen [RT93, Shahrokhi et al. SSSV97, and Székely [S04a]. The embedding method generates a planar drawing (embedding) $D(G)$ of a graph $G$ based on a drawing $D(H)$ of a subgraph $H \subset G$. The drawing $D(G) \operatorname{respects} D(H)$ on the edges of $H$ and for every edge $e=(v, w) \in G \backslash H$, the drawing of $e$ follows "infinitesimally close" to a path between $v$ and $w$ in the drawing $D(H)$. We can distinguish two categories of crossings that involve edges of $G \backslash H$ in the drawing $D(G)$. A first category crossing arises infinitesimally close to a crossing in $D(H)$. A second category crossing arises infinitesimally close to a vertex in $D(H)$.

We illustrate the embedding method with a bound on the minimum decay of the crossing number after deleting one edge. This improves on the RichterThomassen bound for graphs with $m \geq 7.66 n$ edges.

Proposition 1. For every connected graph $G$ with $n$ vertices and $m$ edges, there is an edge $e$ of $G$ such that

$$
\operatorname{cr}(G-e) \geq \frac{p}{p+2}\left(\operatorname{cr}(G)-m+\frac{n}{2}\right),
$$

where $p=\left\lceil\frac{m}{n-1}-1\right\rceil$.

The proof of Proposition 1 follows immediately from Proposition 2 and Lemma 1 below. Nagamochi and Ibaraki NI92 proved the following lemma, which is a slight variant of Mader's theorem, and shows that every graph with $n$ vertices and $m$ edges has a pair of adjacent vertices with at least $\frac{m}{n-1}$ edge-disjoint paths between them. 
Lemma 1 (Mader, Nagamochi and Ibaraki). If $G$ is a graph with $m$ edges and $n$ vertices, then there is an edge $e=(v, w)$ of $G$ such that there are at least $\frac{m}{n-1}-1$ edge-disjoint paths between $v$ and $w$ in $G-e$.

Proof. Delete maximal spanning forests $F_{1}, F_{2}, \ldots, F_{j}$ one after the other until all edges are deleted. If $e=(v, w)$ is an edge of $F_{j}$, then there is a path between $v$ and $w$ in $F_{i}$ for every $i, 1 \leq i \leq j$. Hence, there are at least $j-1$ edge-disjoint paths between $v$ and $w$ that do not pass through $e$. Since each $F_{i}$ is a forest, it has at most $n-1$ edges, and so we have $m \leq j(n-1)$. Substituting, there are at least $\frac{m}{n-1}-1$ edge-disjoint paths between $v$ and $w$ in $G-e$.

Proposition 2. Let $G$ be a connected graph with $n$ vertices and $m$ edges, and $e=(v, w)$ be an edge of $G$ such that there are $p \geq 1$ edge-disjoint paths between $v$ and $w$ in $G-e$. Then

$$
\operatorname{cr}(G) \leq\left(1+\frac{2}{p}\right) \operatorname{cr}(G-e)+m-\frac{n}{2} .
$$

Proof. Let $D$ be a drawing of $G-e$ in the plane with $\operatorname{cr}(G-e)$ crossings. Let $P_{1}, P_{2}, \ldots, P_{p}$ be $p$ edge-disjoint paths between $v$ and $w$. Consider the drawing $D_{j}$ of $G$ in the plane that respects the drawing $D$ of $G-e$ and the edge $e$ follows infinitesimally close to the path $P_{j}$ between $v$ and $w$ with all loops (and selfcrossings) deleted. Let $k_{j}$ be the number of first category crossings in $D_{j}$. Since the paths $P_{1}, P_{2}, \ldots, P_{p}$ are edge-disjoint, the drawings $D_{1}, D_{2}, \ldots, D_{p}$ of $G$ jointly have at most two first category crossings at each crossing of $D$ : at most two crossings between edges of $G-e$ and different drawings of $e$, as depicted in Figure 1(a). Hence,

$$
\sum_{j=1}^{p} k_{j} \leq 2 \operatorname{cr}(G-e) .
$$

Therefore, there is an index $j, 1 \leq j \leq p$, such that $k_{j} \leq 2 \operatorname{cr}(G-e) / p$.

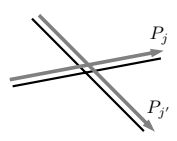

(a)

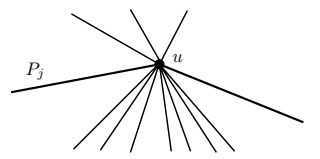

(b)

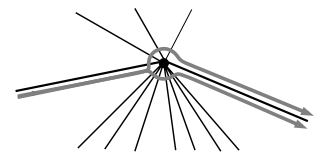

(c)

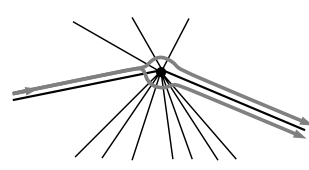

(d)

Fig. 1. Drawings of edge $e$ along two edge-disjoint path $P_{j}$ and $P_{j^{\prime}}$ may give two first category crossings at a crossing of $D$ (a). If a path $P_{j}$ traverses a vertex $u(\mathrm{~b})$, then the edge $e$ drawn along $P_{j}$ can choose between two possible routes around $u(\mathrm{c}-\mathrm{d})$.

At each internal vertex $u$ of a path $P_{j}$, the drawing of $e$ in $D_{j}$ can take two possible routes, as depicted in Figure 1 (c-d). The two possible routes have a total of $\operatorname{deg}(u)-1$ second category crossings at $u$. We draw $e$ along the route with fewer second category crossings, and so there are at most $\frac{1}{2}(\operatorname{deg}(u)-1)$ crossing at 
vertex $u$. Hence, the total number of second category crossings is at most $m-\frac{n}{2}$. Therefore, in the drawing $D_{j}$ of $G$, there are at most $\left(1+\frac{2}{p}\right) \operatorname{cr}(G-e)+m-\frac{n}{2}$ crossings.

The following theorem establishes Theorem 1 for all graphs with $n$ vertices of degree $d_{1}, \ldots, d_{n}$ such that $\operatorname{cr}(G) \geq \frac{7}{16} \sum_{i=1}^{n} d_{i}^{2}$. For graphs that do not satisfy this condition, we alter the proof in Sections 3 and 4

Theorem 2. For every $\epsilon, 0<\epsilon<1$, there is a positive constant $n(\epsilon)$ such that for every $G$ with $n>n(\epsilon)$ vertices, with a degree sequence $d_{1}, \ldots, d_{n}$, and $m>n^{1+\epsilon}$ edges, there is a subgraph $G^{\prime}$ of $G$ with at most $\left(1-\frac{\epsilon}{8}\right) m$ edges such that

$$
4 \operatorname{cr}\left(G^{\prime}\right) \geq \operatorname{cr}(G)-\frac{3}{8} \sum_{i=1}^{n} d_{i}^{2} .
$$

Proof. Erdős and Simonovits [ES82] proved that for every integer $r>1$, there is a constant $c_{r}$ such that every graph $G$ with $n$ vertices and $m>c_{r} n^{1+\frac{1}{r}}$ edges contains a cycle of length $2 r$. This implies that for $0<\epsilon<1$, there is a positive integer $r$ satisfying $\frac{1}{\epsilon}<r \leq \frac{2}{\epsilon}$ so that every sufficiently large graph $G$ with $m>n^{1+\epsilon}$ edges contains a family $\mathcal{C}$ of edge-disjoint cycles of length $2 r$ that cover at least half of the edges of $G$. Let $G^{\prime}$ be a subgraph of $G$ formed by deleting an arbitrary edge $e_{j}$ from each cycle $C_{j} \in \mathcal{C}$. The remaining edges of cycle $C_{j}$ form a path $P_{j}$. Hence, the number of edges of $G \backslash G^{\prime}$ is at least $\frac{\epsilon}{8} m$. Let us denote the vertices of $G$ by $v_{i}, i=1,2, \ldots, n$, such that the degree of $v_{i}$ is $d_{i}$ in $G$ and $d_{i}^{\prime}$ in $G^{\prime}$. Let $h_{i}=d_{i}-d_{i}^{\prime}$, which is the number of edges incident to $v_{i}$ in $G \backslash G^{\prime}$.

Consider a drawing $D^{\prime}$ of $G^{\prime}$ in the plane with $\operatorname{cr}\left(G^{\prime}\right)$ crossings. We generate a drawing $D$ of $G$ based on $D^{\prime}$ by applying the embedding method. In particular, for every edge $e_{j}$ of cycle $C_{j} \in \mathcal{C}$, we draw $e_{j}$ along the path $P_{j}$. Since the paths $P_{j}$ with $C_{j} \in \mathcal{C}$ are edge-disjoint, $D$ has at most 4 crossings at every crossing of $D^{\prime}$. Therefore, the total number of crossings of $D^{\prime}$ and first category crossings of $D$ is at most $4 \mathrm{cr}\left(G^{\prime}\right)$.

Next we estimate the number of second category crossings. Each of the $h_{i}$ edges incident to $v_{i}$ in $G \backslash G^{\prime}$ is drawn, in a neighborhood of $v_{i}$, close to one of the $d_{i}^{\prime}$ edges incident to $v_{i}$ in $G^{\prime}$. The vertex $v_{i}$ with degree $d_{i}^{\prime}$ in $G^{\prime}$ is an internal node of at most $\left\lfloor\left(d_{i}^{\prime}-h_{i}\right) / 2\right\rfloor$ paths $P_{j}$. For every such path $P_{j}$, the edge $e_{j}$ is drawn along one of two possible routes, as depicted in Figure 1(c-d), with the minimum number of crossings with the edges of $G$ incident to the vertex $v_{i}$. Every edge $e_{j} \in G \backslash G^{\prime}$ passing though a small neighborhood of $v_{i}$ has at most $\left\lfloor\left(d_{i}^{\prime}+h_{i}-1\right) / 2\right\rfloor$ second category crossings with edges of $G$ incident to $v_{i}$. Each pair of edges passing through a small neighborhood of $v_{i}$ cross at most once. So the total number of second category crossings at $v_{i}$ is at most

$$
\left\lfloor\frac{d_{i}^{\prime}-h_{i}}{2}\right\rfloor \cdot\left\lfloor\frac{d_{i}^{\prime}+h_{i}-1}{2}\right\rfloor+\left(\begin{array}{c}
\left\lfloor d_{i}^{\prime}-h_{i} / 2\right\rfloor \\
2
\end{array}\right)<\frac{3}{8} d_{i}^{\prime 2} \leq \frac{3}{8} d_{i}^{2} .
$$

Summing over all vertices, we have at most $\sum_{i=1}^{n} \frac{3}{8} d_{i}^{2}$ second category crossings. 
Hence, we have

$$
\operatorname{cr}(G) \leq 4 \operatorname{cr}\left(G^{\prime}\right)+\frac{3}{8} \sum_{i=1}^{n} d_{i}^{2} .
$$

\section{The Sum of Degree Squares and the Crossing Number}

The bisection width, denoted by $b(G)$, is defined for every simple graph $G$ with at least two vertices. $b(G)$ is the smallest nonnegative integer such that there is a partition of the vertex set $V=V_{1} \cup^{*} V_{2}$ with $\frac{1}{3} \cdot|V| \leq V_{i} \leq \frac{2}{3} \cdot|V|$ for $i=1,2$, and $\left|E\left(V_{1}, V_{2}\right)\right| \leq b(G)$. Extending the Lipton-Tarjan separator theorem [LT79], Gazit and Miller [GM90] established an upper bound on the bisection width in terms of the sum of degree squares.

Theorem 3 (Gazit and Miller). Let $G$ be a planar graph with $n$ vertices of degree $d_{1}, d_{2}, \ldots, d_{n}$. Then

$$
b^{2}(G) \leq \frac{5+2 \sqrt{6}}{4} \cdot \sum_{i=1}^{n} d_{i}^{2} .
$$

Pach, Shahrokhi, and Szegedy [PSS96] used Theorem 3 to relate the bisection width with the crossing number.

Theorem 4 (Pach, Shahrokhi, and Szegedy). Let $G$ be a graph with $n$ vertices of degree $d_{1}, d_{2}, \ldots, d_{n}$. Then

$$
40 \mathrm{cr}(G) \geq b^{2}(G)-\frac{5}{2} \cdot \sum_{i=1}^{n} d_{i}^{2}(G) .
$$

Pach, Spencer and Tóth [PST00] have further exploited the connection between the bisection width and the crossing number. They have established lower bounds on the crossing number of graphs with some monotone graph property in terms of the number of edges and vertices of the graph. A simplified version of their proof method yields the following bounds.

Lemma 2. Let $G(V, E)$ be a graph with $n$ vertices of degree $d_{1}, d_{2}, \ldots, d_{n}$, and $m \geq 8 n^{7 / 5} \log ^{2 / 5} n$ edges. Then

$$
\operatorname{cr}(G) \geq \frac{1}{24} \sum_{i=1}^{n} d_{i}^{2} .
$$

This bound is better than the classical lower bound (11) due to Ajtai et al. ACNS82 and Leighton [L84 for graphs of irregular degree patterns and $m=$ $O\left(n^{3 / 2}\right)$ edges. Consider the complete bipartite graph $K_{a, b}$ with $n=a+b$ vertices and $m=a b$ edges, where $a \leq b$. For this graph, our Lemma 2 gives $\operatorname{cr}(G)=$ $\Omega\left(a b^{2}\right)$, which is a tighter than the classical $\Omega\left(m^{3} / n^{2}\right)=\Omega\left(a^{3} b\right)$ bound for $(8+o(1)) b^{2 / 5} \log ^{2 / 5} b \leq a \leq \sqrt{b}$, where the $o(1)$ term goes to 0 as $b \rightarrow \infty$. Similar bounds have also been deduced by Pach, Solymosi, and Tardos [PST06. 
Proof of Lemma 2. We decompose the graph $G$ by the following recursive algorithm into induced subgraphs such that every subgraph is either a singleton or its squared bisection width is at least five times the sum of its degree squares. In an induced subgraph $H \subseteq G$, we denote by $\operatorname{deg}_{H}(v)$ the degree of a vertex $v \in V(H)$.

1. Let $S_{0}=\{G\}$ and $i=0$.

2. Repeat until $|V(H)|=1$ or $b^{2}(H) \geq 5 \sum_{v \in H} \operatorname{deg}_{H}^{2}(v)$ for every $H \in S_{i}$.

Set $i:=i+1$ and $S_{i+1}:=\emptyset$. For every $H \in S_{i}$, do

- If $b^{2}(H) \geq 5 \sum_{v \in H} \operatorname{deg}_{H}^{2}(v)$ or $|V(H)| \leq(2 / 3)^{i}|V|$, then let $S_{i+1}:=$ $S_{i+1} \cup\{H\}$;

- otherwise split $H$ into graphs $H_{1}$ and $H_{2}$ along an edge separator of 3. Return $S_{i}$. size $b(H)$, and let $S_{i+1}:=S_{i+1} \cup\left\{H_{1}, H_{2}\right\}$.

First, we show that the algorithm is correct. In every round, every graph $H \in S_{i}$ that does not satisfy the end condition has at most $|V(H)| \leq(2 / 3)^{i} \cdot|V|$ vertices. The algorithm terminates in $t \leq \log _{(3 / 2)} n$ rounds, and it returns a set $S_{t}$ of induced subgraphs. By Theorem 4 and the end condition of the decomposition algorithm, for every $H \in S_{t}$ we have $40 \mathrm{cr}(H) \geq(5 / 2) \sum_{v \in H} \operatorname{deg}_{H}^{2}(v)$. So

$$
40 \operatorname{cr}(G) \geq 40 \sum_{H \in S_{t}} \operatorname{cr}(H) \geq \frac{5}{2} \cdot \sum_{H \in S_{t}} \sum_{v \in H} \operatorname{deg}_{H}^{2}(v) \geq \frac{5}{2} \cdot \sum_{v \in V} \operatorname{deg}_{H(v, t)}^{2}(v),
$$

where $H(v, i)$ denotes the graph $H \in S_{i}$ containing vertex $v \in V$.

Next, we count the number of edges deleted during the recursive decomposition. Following an argument of [PST00], we count separately the edges deleted in each step of the decomposition algorithm. Let $S_{i}^{\prime}=\left\{H: H \in S_{i}, H \notin S_{i+1}\right\}$, that is, $S_{i}^{\prime}$ consists of those subgraphs in $S_{i}$ that are decomposed at step $i$. Notice that $\left|S_{i}^{\prime}\right|<\left(\frac{3}{2}\right)^{i+1}$ since every subgraph of $S_{i}$ that splits has more than $(2 / 3)^{i+1}|V|$ vertices. Let $V_{i}=\left\{v: v\right.$ is a vertex of a graph $\left.H \in S_{i}^{\prime}\right\}$.

In step $i$, when some of the subgraphs in $S_{i}$ are decomposed in $S_{i+1}$, the total number of deleted edges is at most

$$
\sum_{H \in S_{i}^{\prime}} \sqrt{5 \sum_{v \in H} \operatorname{deg}_{H}^{2}(v)}
$$

Using the Cauchy-Schwartz inequality, we have

$$
\sum_{H \in S_{i}^{\prime}} \sqrt{5 \sum_{v \in H} \operatorname{deg}_{H}^{2}(v)} \leq \sqrt{5\left|S_{i}^{\prime}\right|} \sqrt{\sum_{v \in V_{i}} \operatorname{deg}_{H(v, i)}^{2}(v)} \leq \sqrt{5\left(\frac{3}{2}\right)^{i+1}} \sqrt{\sum_{v \in V_{i}} \operatorname{deg}_{H(v, i)}^{2}(v)} .
$$

Since $|V(H)| \leq\left(\frac{2}{3}\right)^{i}|V|$ for each subgraph $H \in S_{i}^{\prime}$, we conclude that

$$
\begin{aligned}
& \sqrt{5\left(\frac{3}{2}\right)^{i+1}} \sqrt{\sum_{v \in V_{i}} \operatorname{deg}_{H(v, i)}^{2}(v)} \leq \sqrt{5\left(\frac{3}{2}\right)^{i+1}} \sqrt{\max _{v \in V_{i}} \operatorname{deg}_{H(v, i)}(v) \cdot \sum_{v \in V_{i}} \operatorname{deg}_{H(v, i)}(v)} \\
& \leq \sqrt{5\left(\frac{3}{2}\right)^{i+1}} \sqrt{\left(\frac{2}{3}\right)^{i} n(2 m)} \leq \sqrt{15 m n} .
\end{aligned}
$$


Since the algorithm terminates in at $\operatorname{most} \log n / \log (3 / 2)$ steps, the total number of edges deleted throughout the decomposition algorithm is at most

$$
\frac{\sqrt{15}}{\log (3 / 2)} \sqrt{m n} \log n<7 \sqrt{m n} \log n .
$$

If we increase the degree of a vertex by one, the degree square increases by at most $2 n-1<2 n$. By putting back the deleted edges, the sum of degree squares increases by less than $28 m^{1 / 2} n^{3 / 2} \log n$. From Inequality (2), we have

$$
8 \operatorname{cr}(G) \geq 8 \cdot \frac{1024}{31827} \cdot \frac{m^{3}}{n^{2}} \geq 28 m^{1 / 2} n^{3 / 2} \log n,
$$

if $m \geq 8 n^{7 / 5} \log ^{2 / 5} n$. Summing Inequalities (3) and (4), we obtain

$$
24 \operatorname{cr}(G) \geq \sum_{v \in V} \operatorname{deg}_{H(v, t)}^{2}(v)+88 m^{1 / 2} n^{3 / 2} \log n \geq \sum_{i=1}^{n} d_{i}^{2} .
$$

This completes the proof of Lemma 2

We are now ready prove Theorem 1 for the case that $m \geq 8 n^{7 / 5} \log ^{2 / 5} n$.

Theorem 5. For every $\epsilon>0$, there is a constant $n_{0}$ depending on $\epsilon$ such that if $G$ is a graph with $n>n_{0}$ vertices and $m>8 n^{7 / 5} \log ^{2 / 5} n$ edges, then $G$ has a subgraph $G^{\prime}$ formed by deleting at least $m / 20$ edges from $G$ such that $\operatorname{cr}\left(G^{\prime}\right) \geq \frac{1}{13} \operatorname{cr}(G)$.

Proof. Combining Theorem 2 and Lemma 2 we obtain

$$
\operatorname{cr}(G) \leq 4 \operatorname{cr}\left(G^{\prime}\right)+\frac{3}{8} \sum_{i=1}^{n} d_{i}^{2} \leq 4 \operatorname{cr}\left(G^{\prime}\right)+9 \operatorname{cr}\left(G^{\prime}\right)=13 \operatorname{cr}\left(G^{\prime}\right)
$$

\section{Proof of Theorem 1}

Theorem 5 leaves us with the case that $n^{1+\epsilon} \leq m<8 n^{7 / 5} \log ^{2 / 5} n$. Instead of Lemma 4, we employ the following bounds.

Lemma 3. Let $G$ be a graph with $n$ vertices of degree $d_{1}, d_{2}, \ldots, d_{n}$, and $m$ edges. For any $\delta, 0<\delta<1$, let $\Delta=\Delta(\delta)$ be the integer such that $\sum_{i=1}^{n} \min \left(d_{i}, \Delta\right)$ $<2 \delta m$ but $\sum_{i=1}^{n} \min \left(d_{i}, \Delta+1\right) \geq 2 \delta m$. The crossing number of $G$ is bounded by the sum of truncated degree squares. If $m \geq 45(1-\delta)^{-2} n \log ^{2} n$, then

$$
\operatorname{cr}(G) \geq \frac{1}{16} \sum_{i=1}^{n}\left(\min \left(d_{i}, \Delta\right)\right)^{2} .
$$


Lemma 4. Let $G$ be a graph with $n$ vertices and $m$ edges, and let $d_{1} \leq d_{2} \leq$ $\ldots \leq d_{n}$ denote the degree sequence sorted in monotone increasing order. Let $\ell$ be the integer such that $\sum_{i=1}^{\ell-1} d_{i}<4 m / 3$ but $\sum_{i=1}^{\ell} d_{i} \geq 4 m / 3$. The crossing number of $G$ is bounded by a prefix sum of the degree squares. If $m=\Omega\left(n \log ^{2} n\right)$, then

$$
\operatorname{cr}(G) \geq\left(\frac{1}{64}-o(1)\right) \sum_{i=1}^{\ell} d_{i}^{2} .
$$

Proof of Lemma 3. Run the recursive decomposition algorithm described in the previous section on graph $G$. We have shown that during the algorithm at most $\left(\sqrt{15} / \log \frac{3}{2}\right)$

$\sqrt{m n} \log n$ edges are deleted. This is less than $(1-\delta) m$ if $m \geq 45(1-\delta)^{-2} n \log ^{2} n$.

We are now ready to estimate $\sum_{v \in V} \operatorname{deg}_{H(v, t)}^{2}(v)$. Since the number of edges decreased by at most $(1-\delta) m$, the sum of degrees decreased by at most $(2-$ $2 \delta) m$. The sum of degree squares decreases maximally if the highest degrees are truncated to at most $\Delta$, and so we have

$$
\sum_{v \in V} \operatorname{deg}_{H(v, t)}^{2}(v) \geq \sum_{i=1}^{n}\left(\min \left(d_{i}, \Delta\right)\right)^{2} .
$$

This completes the proof of Lemma 3.

Proof of Lemma 4. We extend the argument of the previous proof with $\delta=\frac{5}{6}$. If $d_{\ell} \leq \Delta$, then the right hand side of (5) must clearly be at least $\sum_{i=1}^{\ell} d_{i}^{2}$ and our proof is complete. Let us assume that $\Delta<d_{\ell}$. Refer to Figure 2 .

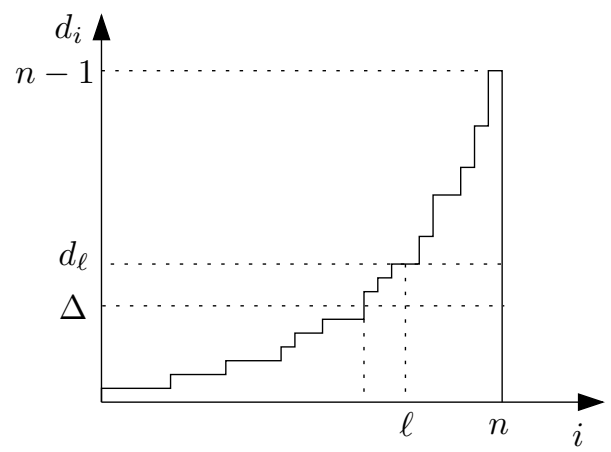

Fig. 2. The monotone increasing degree sequence of a graph $G$

Recall that $\sum_{i=1}^{n} d_{i}=2 m$. We have assumed that $\sum_{i=\ell+1}^{n} d_{i} \leq \frac{2 m}{3}<\sum_{i=\ell}^{n} d_{i}$, and for $\delta=\frac{5}{6}$ we have $\sum_{i=1}^{n} \min \left(d_{i}, \Delta\right)<\frac{5 m}{3} \leq \sum_{i=1}^{n} \min \left(d_{i}, \Delta+1\right)$. It follows that $(n-\ell+1)(\Delta+1)>\frac{m}{3}$. Since $\Delta<n$ and $n=o(m)$, we conclude that $(n-\ell) \Delta>(1-o(1)) \frac{m}{3}$. 
Observe that $(n-\ell) d_{\ell} \leq \sum_{i=\ell+1}^{n} d_{i} \leq \frac{2 m}{3}$, and so $m \geq \frac{3}{2}(n-\ell) d_{\ell}$. Furthermore, observe that $\sum_{i=1}^{\ell} \max \left(0, d_{i}-\Delta\right) \leq \sum_{i=1}^{n} \max \left(0, d_{i}-\Delta\right) \leq n+$ $\sum_{i=1}^{n} \max \left(0, d_{i}-(\Delta+1)\right) \leq n+\frac{m}{3}=(1+o(1)) \frac{m}{3}$. Putting these simple observations together, we obtain

$$
\begin{aligned}
\sum_{i=\ell+1}^{n}\left(\min \left(d_{i}, \Delta\right)\right)^{2} & =(n-\ell) \Delta^{2}>\left(\frac{1}{3}-o(1)\right) m \Delta \geq\left(\frac{1}{2}-o(1)\right)(n-\ell) d_{\ell} \Delta \\
& \geq\left(\frac{1}{6}-o(1)\right) d_{\ell} m \geq\left(\frac{1}{2}-o(1)\right) d_{\ell} \sum_{i=1}^{\ell} \max \left(0, d_{i}-\Delta\right) \\
& \geq\left(\frac{1}{2}-o(1)\right) \sum_{i=1}^{\ell}\left(\max \left(0, d_{i}-\Delta\right)\right)^{2} .
\end{aligned}
$$

We can now estimate the right hand side of Inequality (51).

$$
\begin{aligned}
\sum_{i=1}^{n}\left(\min \left(d_{i}, \Delta\right)\right)^{2} & =\sum_{i=1}^{\ell}\left(\min \left(d_{i}, \Delta\right)\right)^{2}+\sum_{i=\ell+1}^{n}\left(\min \left(d_{i}, \Delta\right)\right)^{2} \\
& \geq \sum_{i=1}^{\ell}\left(\min \left(d_{i}, \Delta\right)\right)^{2}+\left(\frac{1}{2}-o(1)\right) \sum_{i=1}^{\ell}\left(\max \left(0, d_{i}-\Delta\right)\right)^{2} \\
& \geq\left(\frac{1}{2}-o(1)\right) \sum_{i=1}^{\ell}\left(\min \left(d_{i}, \Delta\right)\right)^{2}+\left(\max \left(0, d_{i}-\Delta\right)\right)^{2} \\
& \geq\left(\frac{1}{4}-o(1)\right) \sum_{i=1}^{\ell} d_{i}^{2} .
\end{aligned}
$$

Comparing the above inequality with Inequalities (3) and (5), we obtain $\operatorname{cr}(G) \geq$ $\left(\frac{1}{64}-o(1)\right) \sum_{i=1}^{\ell} d_{i}^{2}$.

We can now prove Theorem 1 in general. Order the vertices $v_{1}, v_{2} \ldots v_{n}$ of $G$ such that their degree sequence $d_{1}, d_{2}, \ldots, d_{n}$ monotone increases. Let $\ell$ be the integer such that $\sum_{i=1}^{\ell-1} d_{i}<\frac{4 m}{3}$ but $\sum_{i=1}^{\ell} d_{i} \geq \frac{4 m}{3}$. Consider the graph $G_{0}$ induced by the vertices $v_{1}, v_{2}, \ldots, v_{\ell}$. Notice that $G_{0}$ has at least $\frac{m}{3}$ edges. We choose a family $\mathcal{C}$ of edge-disjoint cycles of length at most $\frac{4}{\epsilon}$ from $G_{0}$ so that at least half of the edges of $G_{0}$ are covered by cycles of $\mathcal{C}$. Let $G^{\prime}$ be a subgraph of $G$ formed by deleting an edge $e_{j}$ from each cycle $C_{j} \in \mathcal{C}$. We have deleted at least $\frac{1}{2} \cdot \frac{\epsilon}{4} \cdot \frac{m}{3}=\frac{\epsilon}{24} m$ edges. Let $m^{\prime}$ be the number of edges of $G^{\prime}$ and $d_{i}^{\prime}$ be the degree of $v_{i}$ in $G^{\prime}$. We have $d_{i}^{\prime} \leq d_{i}$ for $1 \leq i \leq \ell$ and $d_{i}^{\prime}=d_{i}$ for $i>\ell$. It follows that $\sum_{i=1}^{\ell-1} d_{i}^{\prime}<\frac{4 m^{\prime}}{3}$. By Lemma 4, we have $\operatorname{cr}\left(G^{\prime}\right) \geq\left(\frac{1}{64}-o(1)\right) \sum_{i=1}^{\ell} d_{i}^{\prime 2}$. If we apply the embedding method to draw graph $G$ based on the drawing of $G^{\prime}$ with $\operatorname{cr}\left(G^{\prime}\right)$ crossings and drawing each $e_{j}$ along $P_{j}$, we obtain

$$
\operatorname{cr}(G) \leq 4 \mathrm{cr}\left(G^{\prime}\right)+\frac{3}{8} \sum_{i=1}^{\ell} d_{i}^{\prime 2} .
$$

Hence, we have $\operatorname{cr}(G) \leq 4 \operatorname{cr}\left(G^{\prime}\right)+\frac{3}{8}(64+o(1)) \operatorname{cr}\left(G^{\prime}\right)=(28+o(1)) \operatorname{cr}\left(G^{\prime}\right)$. 


\section{Acknowledgments}

We would like to thank Daniel J. Kleitman, János Pach, Rados Radoičić, and Géza Tóth for helpful comments. Thanks to László Szegő for directing us to Nagamochi and Ibaraki's results.

\section{References}

[ACNS82] M. Ajtai, V. Chvátal, M. Newborn, and E. Szemerédi: Crossing-free subgraphs, in Theory and Practice of Combinatorics, vol. 60 of Mathematical Studies, North-Holland, Amsterdam, 1982, pp. 9-12.

[ES82] P. Erdős and M. Simonovits: Compactness results in extremal graph theory, Combinatorica 2 (1982), 275-288.

[GM90] H. Gazit and G. L. Miller: Planar separators and the Euclidean norm, in SIGAL International Symposium on Algorithms, vol. 450 of LNCS, SpringerVerlag, Berlin, 1990, pp. 338-347.

[L83] T. Leighton: Complexity Issues in VLSI, MIT Press, Cambridge, MA, 1983,

[L84] T. Leighton: New lower bound techniques for VLSI, Math. Systems Theory 17 (1984), 47-70.

[LT79] R. J. Lipton and R. E. Tarjan: A separator theorem for planar graphs, SIAM J. Appl. Math. 36 (1979), 177-189.

[NI92] H. Nagamochi and T. Ibaraki: A linear-time algorithm for finding a sparse $k$-connected spanning subgraph of a $k$-connected graph, Algorithmica 7 (1992), 583-596.

[PRTT04] J. Pach, R. Radoičić, G. Tardos, and G. Tóth: Improving the Crossing Lemma by finding more crossings in sparse graphs, in 20th ACM Symposium on Computational Geometry, ACM Press, New York, 2004, pp. 68-75

[PSS96] J. Pach, F. Shahrokhi, and M. Szegedy: Applications of the crossing number, Algorithmica 16 (1996), 111-117.

[PST06] J. Pach, J. Solymosi, and G. Tardos: Crossing numbers of imbalanced graphs, lecture presented at SIAM Conf. Discrete Math. (Victoria, BC, 2006).

[PST00] J. Pach, J. Spencer, and G. Tóth: New bounds on crossing numbers, Discrete Comput. Geom. 24 (2000), 623-644.

[PT00] J. Pach and G. Tóth: Thirteen problems on crossing numbers, Geombinatorics 9 (2000), 194-207.

[RT93] B. Richter and C. Thomassen: Minimal graphs with crossing number at least $k$, J. Combin. Theory Ser. B 58 (1993), 217-224.

[S00] G. Salazar: On a crossing number result of Richter and Thomassen, $J$. Combin. Theory Ser. B $\mathbf{7 9}$ (2000), 98-99.

[SSSV97] F. Shahrokhi, O. Sýkora, L. A. Székely, and I. Vrt’o: Crossing numbers: bounds and applications, in Intuitive geometry (Budapest, 1995), 179-206, vol. 6 of Bolyai Soc. Math. Stud., János Bolyai Math. Soc., Budapest, 1997.

[S04] L. A. Székely: A successful concept for measuring non-planarity of graphs: the crossing number, Discrete Math. 276 (2004), 331-352.

[S04a] L. A. Székely: Short proof for a theorem of Pach, Spencer, and Tóth, in Towards a theory of geometric graphs, vol. 342 of Contemp. Math., AMS, Providence, RI, 2004, pp. 281-283. 